\title{
Papillary muscle calcification after inferoposterior myocardial infarction
}

\author{
F T Schwender
}

\begin{abstract}
Extensive papillary muscle calcification is quite a rare finding in echocardiographic examinations. A case of a 71 year old man with isolated calcification of the papillary muscles, detected by fluoroscopy and confirmed by echocardiography, is presented. Intracardiac calcifications in patients with prior right coronary artery occlusion and mitral regurgitation should suggest the possibility of posteromedial papillary muscle calcification and dysfunction. (Heart 2001;86:e8)
\end{abstract}

Keywords: papillary muscle; coronary artery disease; calcification

Papillary muscle calcification has been associated with coronary artery disease, dilated cardiomyopathy, mitral valve disease, hypercalcaemia, and increased calcium phosphate product in end stage renal disease..$^{1-3}$ Papillary muscle calcification was first diagnosed radiographically in $1974 .{ }^{4}$ Small calcific deposits are a common finding in elderly people and are located most commonly at the apex. ${ }^{15}$ Extensive papillary muscle calcification is rarely seen.

A 71 year old man with a history of hypertension, myocardial infarction in 1991 and 1994, and three vessel coronary bypass surgery in 1996 presented with an episode of unstable angina. On examination there were no signs of

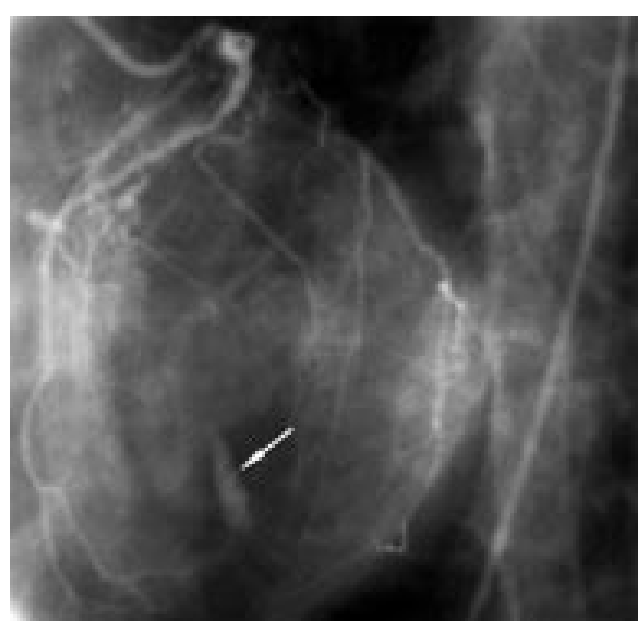

Figure 1 Coronary angiogram showing extensive posteromedial papillary muscle calcification. congestive heart failure but a 3/6 mitral regurgitation murmur was noticed. The patient was started on intravenous heparin, $\beta$ blockers, and aspirin. Serial cardiac enzyme analysis showed no evidence of myocardial infarction.

Given this patient's prior history of a positive stress test in 1999, he underwent cardiac catheterisation and coronary angiography. It showed inferior akinesis, moderate mitral regurgitation, a $70 \%$ proximal stenosis of the left circumflex coronary artery which was heavily calcified, and $100 \%$ occlusion of the obtuse marginal and right coronary arteries. There was a patent venous graft to the distal right coronary artery and a small left internal mammary artery to the left anterior descending artery. Extensive calcification of the posteromedial papillary muscle and moderate calcific deposits of the anteromedial papillary muscle were noticed (fig 1). Review of an echocardiogram taken in 1998 showed an ejection fraction of $45 \%$, normal chamber dimensions, inferior and posterior wall akinesis, and mild to moderate mitral regurgitation. The papillary muscle calcification was confirmed (fig 2). A biochemistry profile showed normal renal function and electrolytes including a normal serum calcium $(8.5 \mathrm{mg} / \mathrm{dl}$ or $2.1 \mathrm{mmol} / \mathrm{l})$ and serum phosphate $(3.2 \mathrm{mg} / \mathrm{dl}$ or $1.0 \mathrm{mmol} / \mathrm{l})$. The patient continued medical treatment and had no further cardiac symptoms three months after discharge. Six months later he presented with ischaemia of the small intestinal and died due to septic complications.

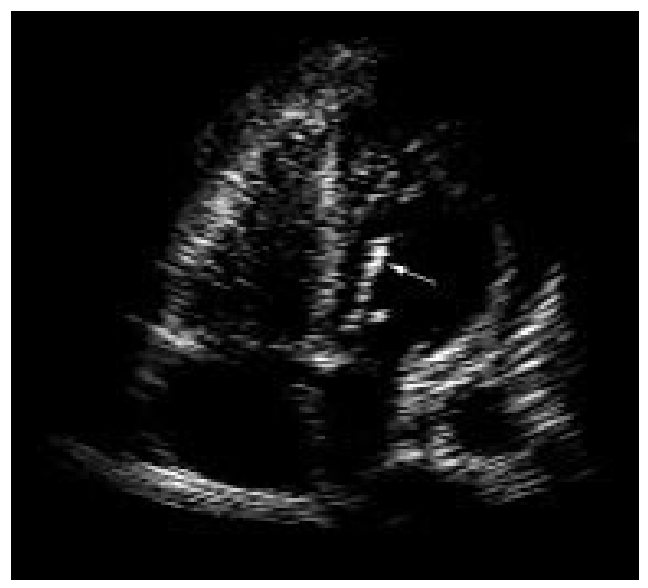

Figure 2 Echocardiogram taken in 1998 confirmed calcification of the posteromedial papillary muscle. 


\section{Discussion}

Calcific deposits are frequently observed in the hearts of people living in the western world. Common locations of calcification are atherosclerotic plaques in the epicardial coronary arteries, followed by the mitral anulus, aortic valve cusps, and apices of the left ventricular papillary muscles. ${ }^{6}$ Extensive papillary muscle calcification is uncommon and only scarce literature about causes and the clinical significance is available. Coronary artery disease has been described in association with papillary muscle calcification. The posteromedial papillary muscle is dependent on its blood supply from the right coronary artery, although sometimes also from a circumflex branch. In contrast, the anteromedial papillary muscle has a dual blood supply. It is believed that in some people the central artery of the papillary muscle is an "end artery" making the muscle prone to necrosis or sclerosis in the case of an ischaemic event. ${ }^{1}$ The patient described had an occlusion of all coronary arteries potentially supplying the posteromedial papillary muscle.

In a study of 17 patients by Come and Riley ${ }^{2}$ an association among mitral regurgitation, congestive heart failure, and papillary muscle calcification was found.
We present a rare case of extensive calcification of the left ventricular papillary muscles diagnosed by fluoroscopy and two dimensional echocardiography. The cause of extensive calcification in this patient is unknown. The finding of intracardiac calcifications in patients with prior right coronary artery occlusion and mitral regurgitation suggests the possibility of post-ischaemic papillary muscle calcification and dysfunction.

1 Madu EC, Cruz IA. The vital role of papillary muscles in mitral and ventricular function: echocardiographic insights. Clin Cardiol 1997;20:93-8.

2 Come PC, Riley MR. M mode and cross-sectional echocardiographic recognition of fibrosis and calcification of the mitral valve chordae and left ventricular papillary muscles. mitral valve chordae and left

3 Ross EM, Rosing DR, Laidlaw JC, et al. Impaired left ventricular systolic and diastolic function without left ventricular dilatation associated with papillary muscle calcification in hypertrophic cardiomyopathy. Am f Cardiol 1986; 57:488-90.

4 Garfein OB, Mau RD, Shimomura S. Cineradiographic recognition of papillary muscle calcification. Chest 1974;66: 207-9.

5 Roberts WC. The senile cardiac calcification syndrome. $A m$ f Cardiol 1986;58:572-4.

6 Roberts WC, Kaufman RJ. Calcification of healed myocardial infarcts. Am f Cardiol 1987;60:28-32. 